\title{
Die Casting Mold Design for Aluminum Alloy Shell of Instrument
}

\author{
Yuanyuan $\mathrm{Li}^{1, \mathrm{a}}$, Meng Wang ${ }^{1}$, Yuchao Guo ${ }^{1}$ \\ ${ }^{1}$ East University of Heilongjiang in Harbin, China
}

\begin{abstract}
This paper is about die casting mold design for aluminum alloy shell of instrument. Three-dimensional model of the casting and mold are designed by using Pro/Engineer and AutoCad which can analyze forming quality. Digital design and theoretical calculation can greatly shorten product development cycle and mold design cycle, improve the accuracy of product design and mold design, and reduce the cost of mold design.
\end{abstract}

\section{Introduction}

Die casting mold is one of the main tools for die casting forming. The level of quality, precision, manufacturing cycle and production efficiency affects the quality, costs and upgrade of products directly. With the appearance of new varieties of alloy and the high request structure of products, mold design process is becoming more and more complex. Using die casting mold CAD/CAM technology in this paper, which fundamentally change the traditional way of product development and mold processing, greatly improve the quality of the product, shorten the development cycle, reduce the production cost.

\section{Surveying and mapping of casting}

The casting for aluminum alloy shell which is made of ZL101 is Surveyed and mapped by vernier caliper. The two-dimensional diagram for shell of instrument is shown in Figure 1.

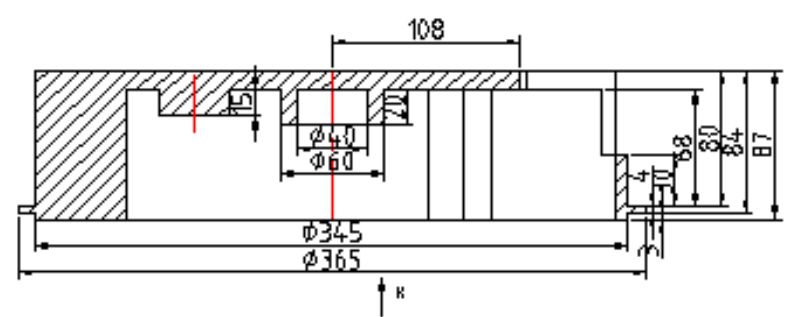

(a)

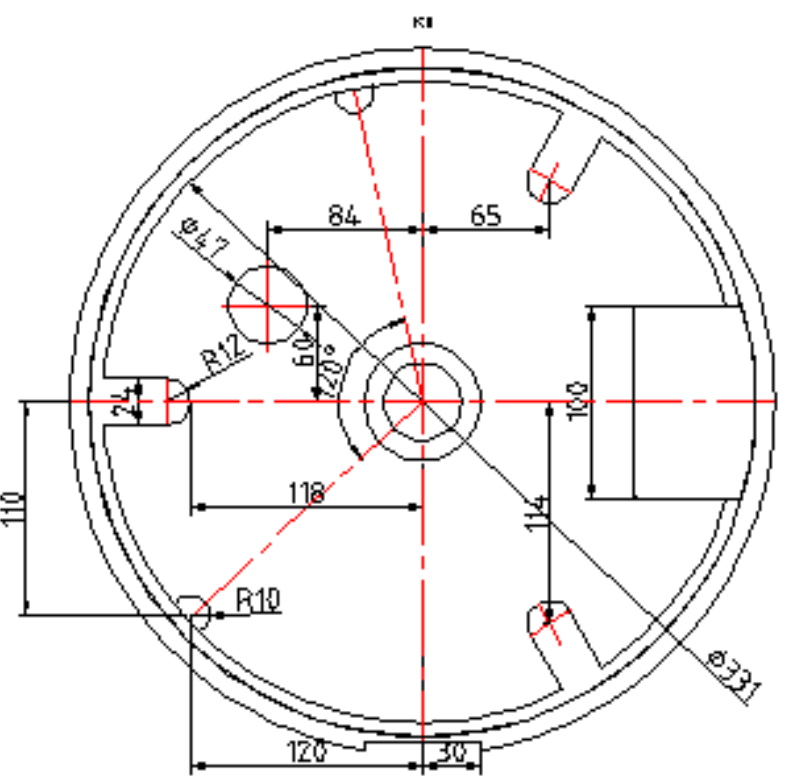

(b)

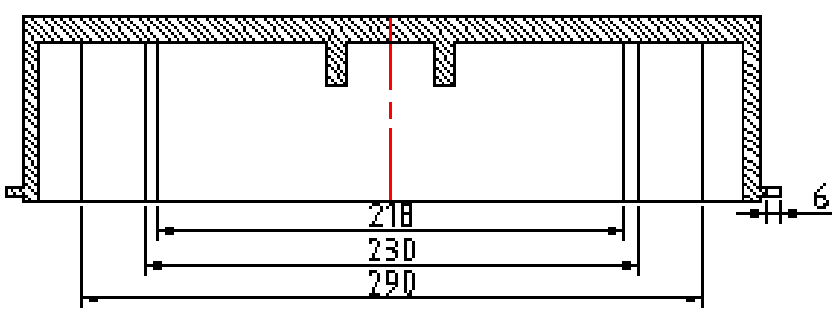

(c)

Figure 1. The two-dimensional diagram for shell of instrument

\section{Selection of the die casting machine}

Using J1113G die casting machine, the parameters are as follows:

\footnotetext{
a Corresponding author: 21870990@qq.com
} 
Die locking force: $1250 \mathrm{kn}$

A * B: $420 * 420$

Injection force: $85-170 \mathrm{kn}$

Pressure chamber diameter: 40 to 60

Injection pressure: $35.5-63.5 \mathrm{MP}$

The largest alloy capacity: aluminum alloy $2.0 \mathrm{~kg}$

The largest projection area of casting: $310 \mathrm{~cm} 2$

Return force of injection punch: $30 \mathrm{kn}$

Range of injection punch: $340 \mathrm{~mm}$

Extension distance of injection punch: $210 \mathrm{~mm}$

Maximum size of mould: $670 * 940 \mathrm{~mm}$

Biggest space of template: $1400 \mathrm{~mm}$

Minimum distance of Template: $600 \mathrm{~mm}$

\section{Selection of mold material}

Structure of die casting mold is complex and there are many parts of mold which play different roles in different places, so that different material should be used in different place. Generally speaking, the material which is used to make die casting mold should have a certain hardness, abrasive resistance, intensity and toughness. And this material should be easy to process. Therefore, we should choose the mold material reasonably according to the requirements of the mold structure, performance, using condition and manufacturing method.

\section{Design of mold structure}

\subsection{Selection of the parting surface}

When parting surface is determined, the following principles need to be obeyed:

A. Parting surface casting should stay in moving die or lower die as far as possible, which can be pushed out easily and the mould structure can also be simplified.

B. The minimalist form of ejection should be considered when the parting surface casting is in moving die.

C. The casting should be under the moving die part or lower die as far as possible, when the casting has side core-pulling.

D. When castings have multiple sets of core-pulling, long side core-pulling should be avoided.

E. Arc parting used in arc head casting can damage the casting appearance. Parting surface should be chosen at the bottom of the head.

F. Generally, the consideration should be given to the appearance of the castings when the parting surface is chosen to avoid the parting marks.

G. Make sure the cavities are in the same parting surface as far as possible when the concentricity has to be considered.

The biggest end of the castings is to be chosen as parting surface, as shown in Figure 2.

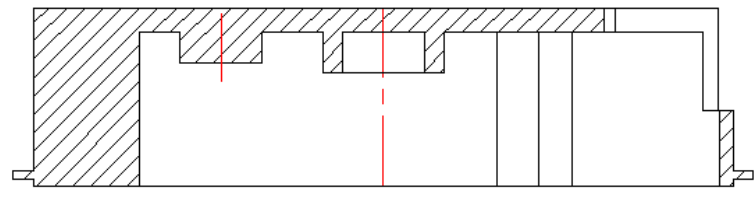

Figure 2. The parting surface

\subsection{Design of gating system}

The liquid metal leaded into the cavity of the channel is called gating system, which is the generic terms of feeding channel starts from the pressure chamber to the gate, includes sprue, cross gate, flow gate and oddments. When designing gating system the following principles should be considered:

A.Gate can not affect the appearance quality of the castings;

B.Gating system should adapt to the forming characteristics in order to ensure the casting quality and molding cycle;

C.The number and the distribution of cavity should be considered when determining the gating system;

D.The shape and size of the molding should be considered when determining the gating system;

E.Using short process in gating system as far as possible to reduce the heat and pressure loss and save raw materials;

F.Gating system should be conducive to exhaust easily to prevent the deformation and displacement of insert core;

Because of the symmetry structure, pin-point gate and sidewall ingate is to be adopted, which is beneficial to uniform flow of liquid metal, fill quickly, and reduce the deformation of the casting. Gating system is shown in Figure 3.

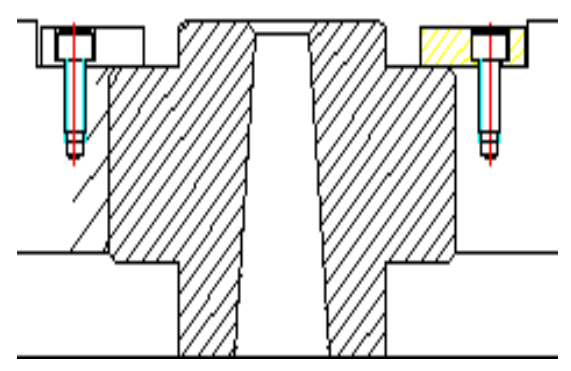

Figure 3. Gating system

Considering the production batch,economic benefits and the accuracy grade of this mold,there are one module and one cavity in this casting. The die casting mold gating system adopts eccentric sprue, and runner in casting is on the side panel, which is good for the flow of metal liquid, rapid filling and reducing the deformation of casting. 


\subsection{Design of ejecting mechanism}

Ejecting mechanism is generally made up of parts, such as push rod, push sleeve, push board and push rod fixed plate. The ejecting mechanism are driven by open mold action of die casting machine, which realize automatic stripping of castings. The casting can stay in the moving mold. Circular push rod is chosen for the casting which has good appearance and reliable structure can avoid deformation or damage. It has the advantages of safety, reliability, simple actions and unfrequent malfunction.

\subsection{Design of guide institutions}

In this design, the guide sleeve is installed on the fixed mold, the guide pin is installed on the moving mold for guiding orientation when clamping. In order to ensure the movement for clamping exactly, two guide pin guide the mold. After mold closing, the location of the push plate directly affects the position of each push rod for putting in place accurately. To ensure the quality of casting, the location of the push plate must be accurate, so four adjustable bolts should be installed between the push plate and the moving mold holder. The location of the push plate can be adjusted by the height of the bolts.

\subsection{Design of overflow system}

In order to let the gas drain out as much as possible by metal liquid, air discharge duct should be set at the site so that liquid metal can be drain out finally. air discharge duct is set behind overflow groove, which can strengthen the effect of overflow and drain.

\subsection{Design of cooling system}

The temperature of mold is controled and adjusted by cooling system. Considering the position of different parts, cooling water channel should be distributed circularly for cooling rapidly.

\subsection{The characteristics of mold structure}

The outstanding characteristic of mold is using the inclined top institutions. Firstly, remove the package tight force of artifacts, then take out the core, which can solve the problem about pulling distance of core, package tight force and huge volume for using a single institution.

\subsection{Analysis and simulation of forming parts}

Three-dimensional entity model of the casting and mold are established through stretching, rotating, scanning, mixing and thin-wall step by using Pro/E. Threedimensional entity model of the casting is shown in Figure 4.

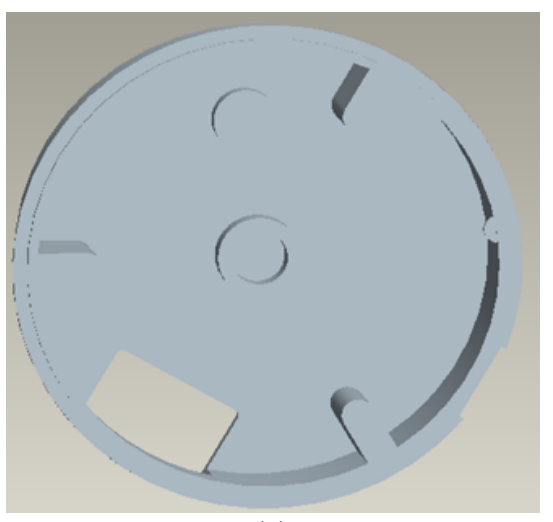

(a)

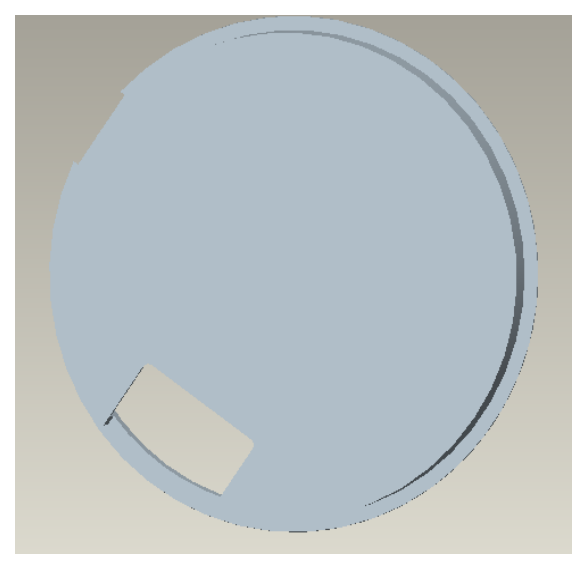

(b)

Figure 4. Three-dimensional entity model of the casting

Numerical control simulation of mold cavity is processed by using mastercam 9.0 after establishing entity model, as shown in Figure 5.

The design steps:

A) Save $3 \mathrm{~d}$ die surface as 09 .igs, and open mastercam9.0;

B) Get into the interface, Select the cplane-side $\rightarrow$ Rotate $\rightarrow$ All $\rightarrow$ Entities $\rightarrow$ Done $\rightarrow$ Origin $\rightarrow$ Generated Rotate the dialog $\rightarrow$ Ok $\rightarrow$ Save as 09. MC9;

C) Go back to the main menu, Create $\rightarrow$ Curve $\rightarrow$ All edges $\rightarrow$ Choose the top plane $\rightarrow$ Done $\rightarrow$ Do it;

D) Go back to the main menu, Create $\rightarrow$ Line $\rightarrow$ Endpoints $\rightarrow$ Sketch $\rightarrow$ Endpoints $\rightarrow$ Draw the line;

E) Go back to the main menu, Xform $\rightarrow$ Translate $\rightarrow$ All $\rightarrow$ Entities $\rightarrow$ Done $\rightarrow$ Between

pts $\rightarrow$ Midpoint $\rightarrow$ Origin $\rightarrow$ Appear translate dialog box $\rightarrow$ Ok;

F) Go back to the main menu, Delete $\rightarrow$ Only $\rightarrow$ Line $\rightarrow$ Choose diagonal $\rightarrow$ Refreshes the screen;

G) Go back to the main menu, Cplane-top $\rightarrow$ Toolpaths $\rightarrow$ Surface $\rightarrow$ Rough $\rightarrow$ Pocket $\rightarrow$ All $\rightarrow$ Surfaces $\rightarrow$ Done. 


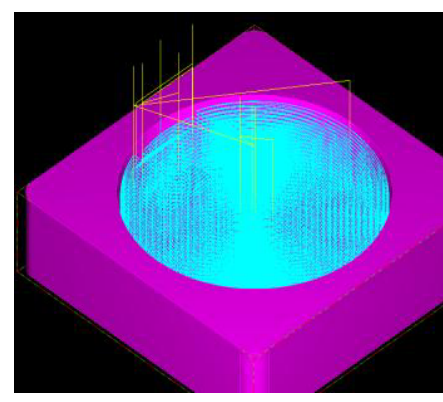

(a)

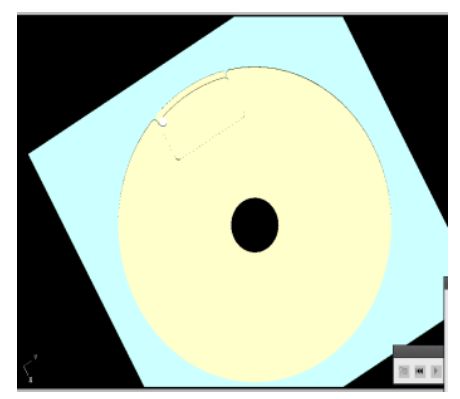

(b)

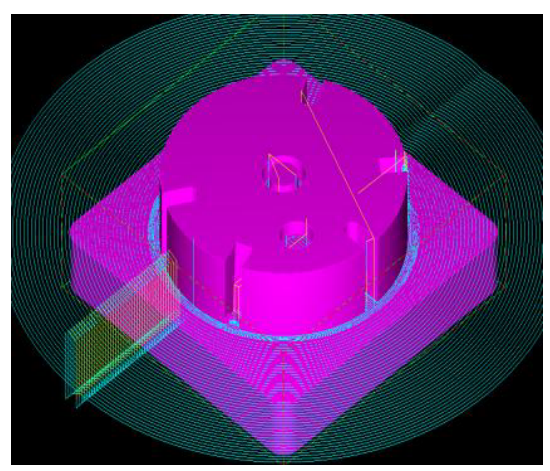

(c)

Figure 5. NC simulation of mold cavity

\section{Conclusions}

This paper is about die casting mold design for aluminum alloy shell of instrument. Three-dimensional model of the casting and mold are designed by using Pro/Engineer and AutoCad which can analyze forming quality. Digital design and theoretical calculation can greatly shorten product development cycle and mold design cycle, improve the accuracy of product design and mold design, and reduce the cost of mold design.

\section{References}

1. Y.K.Woon, K.S.Lee. Development of a die design system for die casting $[\mathrm{J}]$. The International Journal of Advanced Manufacturing Technology. 2004 (5-6).
2. J.C.Choi, T.H.Kwon, J.H.Park, J.H.Kim, C.H.Kim. A Study on Development of a Die Design System for Diecasting $[\mathrm{J}]$. International Journal of Advanced Manufacturing Technology. 2002 (1).

3. S.H.Wu, K.S.Lee, J.Y.H.Fuh. Feature-Based Parametric Design of a Gating System for a DieCasting Die[J]. International Journal of Advanced Manufacturing Technology. 2002 (11).

4. Dr J.C.Lin, C.C.Tai. The runner optimisation design of a die-casting die and the part produced[J]. The International Journal of Advanced Manufacturing Technology. 1998 (2).

5. Roger E.Khayat. A three-dimensional boundary element approach to confined free-surface flow as applied to die casting[J]. Engineering Analysis with Boundary Elements. 1998 (2).

6. Srinivasan A, Pillai U T S, John V, et al. Lowpressure casting of LM25 (Al-7Si-0.3Mg) aluminum alloy[J]. Materials and Manufacturing Processes. 2005, 20(2): 221-230.

7. Zhang B, Cockcroft S L, Maijer D M, et al. Casting defects in low-pressure die-cast aluminum alloy wheels[J]. JOM. 2005, 57(11): 36-43.

8. Bonollo F, Urban J, Bonatto B, et al. Gravity and low pressure die casting of aluminum alloys: A technical and economical benchmark[J]. Metallurgia Italiana. 2005, 97(6): 23-32. 